Bull. Austral. Math. Soc.

Vol. 51 (1995) [145-151]

\title{
ESTIMATING MAHLER'S MEASURE
}

\section{G.R. EvEREST}

In 1962, Mahler defined a measure for integer polynomials in several variables as the logarithmic integral over the torus. Many results exist about the values taken by the measure but many unsolved problems remain. In one variable, it is possible to express the measure as an effective limit of Riemann sums. We show that the same is true in several variables, using a non-obvious parametrisation of the torus together with Baker's Theorem on linear forms in logarithms of algebraic numbers.

0.

Suppose $Q(\underline{z})$ denotes a polynomial in $\mathbf{Z}\left[z_{1}, \ldots, z_{n}\right]$. In 1962, Mahler [6] proposed the following definition of measure (or height) for $Q$;

$$
m(Q)=\int_{0}^{1} \cdots \int_{0}^{1} \log \left|Q\left(e^{2 \pi i \nu_{1}}, \ldots, e^{2 \pi i \nu_{n}}\right)\right| d \nu_{1} \ldots d \nu_{n} .
$$

There exist some beautiful results and some intriguing conjectures about the values of this measure. It is known (see [2,7]) that it vanishes if and only if $Q$ is a monomial multiplied by a product of generalised cyclotomic polynomials. In the case where $n=1$, Lehmer (see [4]) suspected that the non-zero values of the measure are bounded below by a positive constant. He asked whether the smallest measure belongs to the polynomial $x^{10}+x^{9}-x^{7}-x^{6}-x^{5}-x^{4}-x^{3}+x+1$. Sixty years on, this question is unresolved.

Some non-zero values were calculated by Smyth [7]. For example,

$$
m\left(1+x_{1}+x_{2}\right)=\frac{3 \sqrt{3}}{4 \pi} \sum_{n=1}^{\infty}\left(\frac{n}{3}\right) \frac{1}{n^{2}}, \quad m\left(1+x_{1}+x_{2}+x_{3}\right)=\frac{7}{2 \pi^{2}} \zeta(3) .
$$

In (2), $\left(\frac{}{3}\right)$ denotes the Legendre symbol mod 3 and $\zeta$ denotes the Riemann zeta function. The basic method here is an extended version of Jensen's formula. Boyd [2] used the same method to prove formulae such as the following,

$$
\lim _{N \rightarrow \infty} m\left(x_{1}, x_{1}^{N}\right)=m\left(x_{1}, x_{2}\right)
$$

Received 26th April, 1994

Copyright Clearance Centre, Inc. Serial-fee code: 0004-9729/95 \$A2.00+0.00. 
Spurred on by this, he made some conjectures about the closure of the set of values of the measure in several variables in an attempt to see Lehmer's question in a wider context. Recently [5], Mahler's measure has been recognised as defining the entropy for commuting automorphisms of compact groups.

Since there is so much interest in the values taken by the measure we concern ourselves with the more fundamental question of how these may be approximated effectively. Our longer view is theoretical, to try to explain results such as (3). (See comment 3 after the proof of the theorem.) In one variable, write $Q(x)=a \Pi_{j}\left(x-\alpha_{j}\right)$ then, again by Jensen's formula,

$$
m(Q)=\log |a|+\sum_{j} \max \left\{0, \log \left|\alpha_{j}\right|\right\}
$$

This requires a priori knowledge of the roots. Also, it gives no help with the many variable case.

Alternatively, it is possible to express the measure as a limit of Riemann sums. Let $N$ denote a large positive integer, with $\zeta$ denoting a primitive $N$ th root of unity. Then it is possible to realise the following asymptotic formula;

$$
N^{-1} \sum_{j=1}^{N} \log \left|Q\left(\zeta^{j}\right)\right|=m(Q)+O\left(\log N \cdot N^{-A_{1}}\right)
$$

In (5), the formula is effective in the sense that both $0<A_{1}=A_{1}(Q)<1$ and the constant implied by the big $\mathrm{O}$ notation are effectively computable. Moreover, they depend upon the degree of $Q$ and a more primitive version of height such as the absolutely largest coefficient. Of course, we omit from consideration the finitely many zeros of $Q$. The proof of formula (5) depends upon Gelfond's Theorem, namely a lower bound,

$$
N^{-1 / A_{1}}<\left|Q\left(\zeta^{j}\right)\right|
$$

for all $j=1, \ldots, N$, where $A_{1}>0$ is effective. The powers of $\zeta$ are uniformly distributed on $T$, the unit circle and (6) gives a bound of $O\left(N^{-A_{1}}\right)$ for the discrepancy. (We shall assume familiarity with the standard definitions and results from the metrical theory of numbers as in [3].) Therefore, if $Q$ has no zeros on $T$ then $\log |Q|$ is continuous and we may apply Koksma's Theorem to deduce that the Riemann sum differs from the integral by the bound shown in (5). If $Q$ does have zeros on $T$ then we may surround them by $\varepsilon$-neighbourhoods, where $\varepsilon=N^{-1 / A_{1}}$. Precisely, delete from $T$ those $t$ with $|Q(t)|<\varepsilon$ and call the deleted set $T(\varepsilon)$. Applying Koksma again gives the same formula as in (5), except that the integral is adrift. The resulting bound for the error can be calculated by multiplying a bound for the integrand, namely $|\log \varepsilon|$, by a bound for the measure of $T(\varepsilon)$, namely $O\left(N^{-1 / A_{1}}\right)$. 
This method seems to be strangely resistant to generalisation to the many variable case. (See Comment 1 after the proof of the Theorem.) Not the least of our troubles is the absence of a many variable version of Gelfond's Theorem. The aim of this paper is to show that Mahler's measure can be approximated effectively by Riemann sums, using a different parametrisation of the torus together with Baker's Theorem on linear forms in logarithms of algebraic numbers (see [1]). Suppose $\theta$ denotes an algebraic number of absolute value 1 which is not a root of unity. The powers $\theta^{x}$ for $x \in N, \quad 0 \leqslant x<X$ are dense and uniformly distributed in T. Moreover, Baker's Theorem gives a good effective bound for the discrepancy. Using the same kind of reasoning as above, we may show;

$$
X^{-1} \sum_{x<X} \log \left|Q\left(\theta^{x}\right)\right|=m(Q)+O\left(X^{-A_{2}}\right)
$$

for some effective $0<A_{2}<1$, depending upon $Q$ and the degree and the height of $\theta$. Again, in (7), the zeros of $Q$ are ignored. The possibility exists to generalise this method.

As a first guess, choose a vector $\underline{\theta}=\left(\theta_{1}, \ldots, \theta_{n}\right)$ of algebraic numbers with $\left|\theta_{i}\right|=1$ for all $1 \leqslant i \leqslant n$ and let $\underline{x} \in \mathbf{N}^{n}$ with | | denoting the 'max'-norm. Then form the Riemann sum;

$$
X^{-n} \sum_{|\underline{x}|<X} \log \left|Q\left(\theta_{1}^{x_{1}}, \ldots, \theta_{n}^{x_{n}}\right)\right|
$$

Suppose we try to proceed as above by first specialising $n-1$ of the variables. The dependence of $A_{2}$ in (7) upon the resulting polynomial is now a real stumbling block. Instead, we choose algebraic numbers $\theta_{i}, \psi_{i}, 1 \leqslant i \leqslant n$ in such a way that $\left\{1, \theta_{i}, \psi_{i}\right\}$ are multiplicatively independent for each $1 \leqslant i \leqslant n$. (See Note 2 after the proof of the theorem concerning this choice.) Then form the Riemann sum,

$$
S(Q, X, \underline{\theta}, \underline{\psi})=X^{-2 n} \sum_{|(\underline{x}, \underline{y})|<X} \log \left|Q\left(\theta_{1}^{x_{1}} \psi_{1}^{y_{1}}, \ldots, \theta_{n}^{x_{n}} \psi_{n}^{y_{n}}\right)\right|
$$

\section{THEOREM .}

There is an asymptotic formula, as $X \rightarrow \infty$ :

$$
S(Q, X, \underline{\theta}, \underline{\psi})=2^{n} m(Q)+O\left(X^{-A_{3}}\right)
$$

The constants $0<A_{3}<1$ and that implied by the big $O$ notation in (10) are effectively computable and they depend upon $\underline{\theta}, \underline{\psi}$ and $Q$.

The remainder of the paper is occupied with the proof of this theorem. 
1.

Firstly, notice that the number of zeros of $Q(\underline{x}, \underline{y})$ with $|(\underline{x}, \underline{y})|<X$ is $O\left(X^{2 n-1}\right)$ effectively so they will be ignored throughout the remainder of the paper. Given $Q \in \mathbf{Z}\left[z_{1}, \ldots, z_{n}\right]$, we aim to decompose the sum in (10) as follows. We shall determine $\varepsilon_{1}(X)$ such that the sum contains no terms with $|Q(\underline{x}, \underline{y})|<\varepsilon_{1}(X)$ and $|(\underline{x}, \underline{y})|<X$. Begin with the following elementary estimate; there are constants $C_{i}=C_{i}(Q, \underline{\theta}, \underline{\psi})$, $i=1,2$ such that

$$
\frac{C_{1}}{C_{2}^{|\underline{x}|}}<|Q(\underline{x})| .
$$

To see (11), note that the denominator of $Q(\underline{x}, \underline{y})$ is clearly of the form of the left hand side of (11). A bound for the non-zero values of the numerator is obtained by taking the norm from $\mathbf{Q}\left(\theta_{i}, \psi_{j}\right)$ down to $\mathbf{Q}$. This yields a lower bound of the desired shape. Let $\varepsilon_{1}(X)$ denote the smallest value of the left-hand side of $(11)$ as $(\underline{x}, \underline{y})$ run over all elements of $\mathrm{N}^{2 n}$ with $|(\underline{x}, \underline{y})|<X$. Obviously, $\left|\log \varepsilon_{1}\right|=O(X)$, effectively.

Now $(\underline{x}, \underline{y})$ runs over a product of intervals $I_{Q}$ and we consider $Q$ as defined on the real points. Then $Q$ is periodic in each variable with period equal to the corresponding interval. Consider the pre-image in $I_{Q}$ of the inequality $|Q(\underline{x}, \underline{y})| \geqslant \varepsilon_{1}$. Label this $I_{Q}\left(\varepsilon_{1}\right)$. Then $I_{Q}\left(\varepsilon_{1}\right)$ is a compact subset of $I_{Q}$. Moreover, consider each $(\underline{x}, \underline{y}) \in \mathrm{N}^{2 n}$ $\bmod I_{Q}$. The resulting images with $|(\underline{x}, \underline{y})|<X$ are dense and uniformly distributed in $I_{Q}\left(\varepsilon_{1}\right)$. Therefore the function $\log |Q|$ is continuous on the compact set $I_{Q}\left(\varepsilon_{1}\right)$ and we may apply Koksma's Theorem to deduce that

$$
X^{-2 n} \sum_{\substack{|(\underline{x}, \underline{y})|<X,|Q(\underline{x}, \underline{y})| \geqslant \varepsilon_{1}}} \log |Q(\underline{x}, \underline{y})|=\int_{I_{Q}\left(\varepsilon_{1}\right)} \log |Q| d \mu+O\left(\left|\log \varepsilon_{1}\right| D_{X}\right) .
$$

In (12), $D_{X}$ denotes the discrepancy of the sequence $(\underline{x}, \underline{y}) \bmod I_{Q}\left(\varepsilon_{1}\right)$ for $(\underline{x}, \underline{y}) \in \mathrm{N}^{2 n}$ with $|(\underline{x}, \underline{y})|<X$. Also, $\mathrm{d} \mu$ denotes the usual measure giving the Riemann integral on $I_{Q}$. Writing $\theta_{i}^{x_{i}} \psi_{i}^{y_{i}}=\exp \left(x_{i} \log \theta_{i}+y_{i} \log \psi_{i}\right)$, our attention turns to the sequence $x_{i} \log \theta_{i}+y_{i} \log \psi_{i} \bmod 2 \pi i$ with $\left|x_{i}, y_{i}\right|<X$. Baker's Theorem gives an effective lower bound of the form $X^{-1 / C_{3}}$ for the absolute values of this sequence. We obtain an effective bound for the discrepancy of $O\left(X^{-C_{3}}\right)$, where $0<C_{3}=C_{3}(\underline{\theta}, \underline{\psi})<1$.

Simply replacing $\log \varepsilon_{1}$ by the value $O(X)$ obtained above and using $D_{X}$ $=O\left(X^{-C_{3}}\right)$ gives a useless error term, much bigger than the main term. So we practise the following refinement. Let $n-1$ of the $z_{i}$ be determined (say for $2 \leqslant i \leqslant n$ ), corresponding to a specialisation of $2 n-2$ of the pairs $x_{i}, y_{i}$ in the range $\left|x_{i}\right|,\left|y_{i}\right|<X$. 
The result is a polynomial $Q\left(z_{1}\right)$ which we regard also as an exponential polynomial in $x_{1}, y_{1}$. We shall now determine $\varepsilon_{2}=\varepsilon_{2}(X)$ such that the number of solutions $x_{1}, y_{1}$ with $\left|x_{1}\right|,\left|y_{1}\right|<X$ and $\left|Q\left(x_{1}, y_{1}\right)\right|<\varepsilon_{2}$ is bounded by a number $C_{4}=C_{4}(Q)$ which depends on $Q$ but is independent of the specialisation of the other variables. Given $z_{2}, \ldots, z_{n}$ let $Q\left(z_{1}\right)=a \Pi\left(z_{1}-\alpha_{j}\right)$ denote the factorisation of $Q\left(z_{1}\right)$. We ask how it is possible for $\left|Q\left(x_{1}, y_{1}\right)\right|$ to be less than $\varepsilon_{2}$.

Of course this happens only if at least one of the factors of $Q\left(z_{1}\right)$ satisfies the same inequality apart from $\varepsilon_{2}^{1 / K}$ replacing $\varepsilon_{2}$, where $K$ denotes some bound on the multi-degree of $Q$. If it is $|a|<\varepsilon_{2}^{1 / K}$ then induction gives the result we desire. On the other hand, $K+1$ distinct solutions of the inequality $\left|Q\left(z_{1}\right)\right|<\varepsilon_{2}$ will force

$$
\left|\theta_{1}^{x_{1}} \psi_{1}^{y_{1}}-\alpha_{j}\right|<\varepsilon_{2}^{1 / K}, \quad\left|\theta_{1}^{x_{1}^{\prime}} \psi_{1}^{y_{1}^{\prime}}-\alpha_{j}\right|<\varepsilon_{2}^{1 / K},
$$

for some $j$, with $0<x_{1} \neq x_{1}^{\prime}, y_{1} \neq y_{1}^{\prime}<X$. Eliminating $\alpha_{j}$ gives rise to an inequality as follows;

$$
\left|\theta_{1}^{x_{1}} \psi_{1}^{y_{1}}-\theta_{1}^{x_{1}^{\prime}} \psi_{1}^{y_{1}^{\prime}}\right|<2 \varepsilon_{2}^{1 / K},
$$

A simple application of Baker's Theorem shows (13) is impossible for $\varepsilon_{2}$ of the form $X^{-C_{5}}$ for a positive constant $C_{5}$ which depends on $\theta_{1}$ and $\psi_{1}$. Therefore, by induction, taking account of all specialisations, there are constants as claimed and we now record the statement in the following;

Lemma. There are constants $C_{5}=C_{5}(\underline{\theta}, \psi)$ and $C_{6}=C_{6}(Q)$ with the property that the number of solutions of the inequalities

$$
|Q(\underline{x}, \underline{y})|<X^{-C_{8}}, \quad|(\underline{x}, \underline{y})|<X,
$$

is bounded by $C_{6}$.

Using the lemma we can now complete our analysis leading to the proof of the theorem. Let $\varepsilon_{2}$ denote the quantity $X^{-C_{5}}$. Subdivide the intervals in $I_{Q}\left(\varepsilon_{i}\right), i=1,2$ according to the values of $\varepsilon_{i}$. Deal first with the sum,

$$
X^{-2 n} \sum_{\substack{|(\underline{x}, \underline{y})|<X, \varepsilon_{2} \leqslant|Q(\underline{x}, \underline{y})|}} \log |Q(\underline{x}, \underline{y})| .
$$

By Koksma's Theorem, this is the integral

$$
\int_{I_{Q}\left(e_{2}\right)} \log |Q| d \mu
$$


up to an effective error which is

$$
O\left(\left|\log \varepsilon_{2}\right| D_{X}\right)=O\left(\log X \cdot X^{-C_{3}}\right)
$$

upon substituting the values for $D_{X}$ (as used in (12)) and $\varepsilon_{2}$. The integral in (16) differs from the following integral;

$$
\int_{I_{Q}} \log |Q| d \underline{x}
$$

by an amount which is $O\left(\right.$ meas $\left.\left(I_{Q}-I_{Q}\left(\varepsilon_{2}\right)\right)\left|\log \varepsilon_{2}\right|\right)$, where meas denotes the measure on the torus $T^{2 n}$. We claim that this measure is bounded by $\varepsilon_{2}^{C_{7}}$, for some effective constant $C_{7}=C_{7}(Q, \underline{\theta}, \underline{\psi})>0$. Assuming this, we have shown that the sum in (15) differs from the integral in (18) by an effective bound which is $O\left(X^{-C_{8}}\right)$, where $C_{8}=C_{8}(Q, \underline{\theta}, \psi)$. The integral in (18) differs from $m(Q)$ by the appropriate winding number, namely $2^{n}$, which explains the presence of that term in (10).

To prove the claim about the measure, specialise $2 n-1$ of the variables to leave an exponential polynomial, say $Q\left(x_{1}\right)=a \Pi_{j}\left(\theta_{1}^{x_{1}}-\alpha_{j}\right)$. Those specialisations with $a=0$ already have measure zero. As before, a bound of the kind $\left|Q\left(x_{1}\right)\right|<\varepsilon_{2}$ forces a similar bound for a least one of the factors, with $\varepsilon_{2}^{1 / K}$ replacing $\varepsilon_{2}$. If this is $|a|<\varepsilon_{2}^{1 / K}$ then induction gives the result. If it is $\left|\theta_{1}^{x_{1}}-\alpha_{j}\right|<\varepsilon_{2}^{1 / K}$ then clearly $x_{1}$ is determined to within a window proportional to a power of $\varepsilon_{2}$, depending upon $\theta_{1}$ but independent of $\alpha_{j}$. The same is true for all of the variables and concludes the proof of the claim about the measure.

Finally, deal with the sum,

$$
X^{-2 n} \sum_{\substack{|(\underline{x}, \underline{y})|<X, e_{1}<|Q(\underline{x}, \underline{y})| \leqslant e_{2}}} \log |Q(\underline{x}, \underline{y})| .
$$

By the remarks above, if we specialise $2 n-2$ of the variables, there exist only finitely many possibilities for the remaining variables. For these, we can only take the most pessimistic view for the value of $|Q(\underline{x}, \underline{y})|$, namely $\varepsilon_{1}$. Thus the bound for the sum in (19) is the effective estimate $O\left(X^{1-2 n}\right)$, multiplied by the number of specialisations possible, a total (effective) error of $O\left(X^{-1}\right)$.

\section{Comments.}

1. The reason that we could not apply a similar technique with roots of unity is because the multiplicative independence criterion would not be met. We could try to overcome this by choosing the roots to have orders which are coprime. The difficulty then arises of finding a good analogue of the elementary estimate (11). 
2. It is desirable to choose $\theta_{i}, \psi_{i}$ in such a way as to minimise the exponents in the error terms. These depend upon both the degrees and the heights of the numbers. It is not clear yet, whether it is better to choose numbers of small degree such as $(3+4 i) / 5$, given by Pythagorean triples or to choose numbers of small height, such as the Salem numbers.

3. The approximation of $m(Q)$ by Riemann sums of the kind in (9) gives some insight into formula (3). Perhaps this technique will yield an alternative proof or even better, a way of understanding the closure of the set of values of the measure. (See [2] for some conjectures on this topic.)

\section{REFERENCES}

[1] A. Baker (Editor), New advances in transcendence theory (Cambridge University Press, Cambridge. London, New York, Melbourne, 1988).

[2] D.W. Boyd, 'Speculations concerning the range of Mahler's measure', Canad. Math. Bull. 24 (1981), 453-469.

[3] L. Kuipers and H. Niedderreiter, Uniform distribution of sequences (Wiley, 1974).

[4] D.H. Lehmer, 'Factorization of certain cyclotomic functions', Ann. of Math. 34 (1933), 461-479.

[5] D. Lind, K. Schmidt and T. Ward, 'Mahler measure and entropy for commuting automorphisms of compact groups', Invent. Math. 101 (1990), 593-629.

[6] K. Mahler, 'On some inequalities for polynomials in several variables', J. London Math. Soc. 37 (1962), 341-344.

[7] C.J.Smyth, 'A Kronecker-type theorem for complex polynomials in several variables', Canad. Math. Bull. 24 (1981), 447-452.

School of Mathematics

University of East Anglia

Norwich NR4 7TJ

England

e-mail: g.everest@uea.ac.uk 